\section{PM0-246 URINARY MATRIX METALLOPROTEINASES DO NOT CORRELATE WITH ENDOSCOPIC OR HISTOLOGICAL DISEASE ACTIVITY IN ULCERATIVE COLITIS}

\section{doi:10.1136/gutjnl-2012-302514b.246}

${ }^{1} \mathrm{D} S$ Pearl, ${ }^{*}{ }^{2} \mathrm{~S}$ Edgar, ${ }^{3} \mathrm{M}$ Whittaker, ${ }^{3} \mathrm{H}$ Nitch-Smith, ${ }^{2} \mathrm{~J} \mathrm{~F} \mathrm{Brown,}{ }^{2} \mathrm{~J} \mathrm{~K}$ Shute, ${ }^{1} \mathrm{~T}$ M Trebble. 'Department of Gastroenterology, Portsmouth Hospitals NHS Trust, Portsmouth, UK; ${ }^{2}$ Institute of Biomedicine and Biomolecular Sciences, University of Portsmouth, Portsmouth, UK: ${ }^{3}$ Histopathology, Portsmouth Hospitals NHS Trust, Portsmouth, UK

Introduction Ulcerative colitis (UC) is a colonic inflammatory disorder of unconfirmed aetiology. Clinical assessment involves invasive endoscopic examination with a small yet significant procedural risk, on which therapeutic decisions are made. Noninvasive biomarkers may be better tolerated and reduce procedural costs and risks. Matrix metalloproteinases (MMP) are enzymes involved in tissue remodelling; MMP are elevated in mucosa and urine of children with active UC. We measured urinary MMP activity in adult patients with UC, matched controls investigated for functional symptoms and normal healthy volunteers to evaluate as a potential biomarker of disease activity.

Methods Ethical approval and informed consent were obtained. Patients with UC and age-sex matched controls, identified during outpatient assessment, were prospectively recruited and flexible sigmoidoscopy (FS) performed. Endoscopic (Sutherland) and histological (Gomes) appearance in patients with UC was graded. A group of healthy volunteers were recruited at a local University. Urine samples, snap frozen at collection in liquid nitrogen, were thawed, centrifuged, and assayed using commercially obtained fluorescein-labelled gelatinase activity kits. MMP activity was corrected for creatinine concentration. Results were expressed as median \pm IOR. Statistical tests included Kruskal-Wallis analysis and Spearman's correlation.

Results 80 active and 16 quiescent UC patients, 77 age-sex matched controls and 22 normal healthy volunteers were compared. Urinary MMP activity in active compared with quiescent UC $(p=0.185)$ and in each compared with age-sex matched controls $(p=0.237, p=0.525$ respectively) was not significantly different. Exclusion of patients taking 5-aminosalicylates and corticosteroids did not alter significance. There was no correlation between urinary MMP activity and UC disease activity measured endoscopically $(\mathrm{r}=0.09, \mathrm{p}=0.425)$ or histologically $(r=0.178, p=0.127)$. Urinary MMP activity in healthy volunteers was significantly lower than patients with active UC $(p<0.0001)$, quiescent UC $(p<0.002)$, and controls $(p<0.0001)$.

Conclusion Contrary to previously published work, our findings suggest that urinary MMP activity, measured using fluoresceinlabelled gelatinase assay, does not discriminate between quiescent and active UC and does not correlate with UC disease activity. Significant differences noted between healthy volunteers and patients with UC were unexpected, but may reflect difference in group demographics. MMP gelatinase assays are therefore a poor non-invasive biomarker of disease activity in UC.

Competing interests None declared.

\section{PM0-247 MUCOSAL CYTOKINE EXPRESSION IN ULCERATIVE COLITIS: ELEVATED IL-8 BUT NOT TNF- $\alpha$ AND REDUCED TGF- $\beta$ IN INFLAMED COMPARED TO NON-INFLAMED MUCOSA}

doi:10.1136/gutjnl-2012-302514b.247

${ }^{1} \mathrm{D} S \mathrm{P}$ Pearl, ${ }^{*}{ }^{2} \mathrm{~K}$ Shah, ${ }^{3} \mathrm{M}$ Whittaker, ${ }^{3} \mathrm{H}$ Nitch-Smith, ${ }^{2} \mathrm{~J} \mathrm{~F}$ Brown, ${ }^{2} \mathrm{~J} \mathrm{~K}$ Shute, ${ }^{1} \mathrm{~T}$ M Trebble. 'Department of Gastroenterology, Portsmouth Hospitals NHS Trust, Portsmouth, UK; ${ }^{2}$ Institute of Biomedicine and Biomolecular Sciences, University of
Portsmouth, Portsmouth, UK; ${ }^{3}$ Histopathology, Portsmouth Hospitals NHS Trust, Portsmouth, UK

Introduction Ulcerative colitis (UC) is a chronic inflammatory condition of the colon of unconfirmed aetiology. Microscopic examination of inflamed biopsies is characterised by progressive neutrophil infiltration and tissue destruction. There is conflicting evidence from studies on the relative roles of TNF $\alpha$, IL-8, TGF $\beta$ and other cytokines in UC; however, current therapy includes cytokine targeted interventions. We compared cytokine profiles of inflamed and non-inflamed mucosa in patients with distal UC, and age-sex matched controls.

Methods Ethical approval was obtained. Patients were prospectively recruited from outpatients' clinics. Mucosal biopsies at flexible sigmoidoscopy (FS) were taken from UC patients within macroscopically inflamed and non-inflamed proximal mucosa, and from age-sex matched controls undergoing FS. Severity of endoscopic (Sutherland) and histological (Gomes) inflammation were recorded. Quantitative cytokine analysis for IL-4, TNF $\alpha$, IL-17A, IL-8, IL-10, TGF $\beta$ and IFN- $\gamma$ were carried out using commercially available assays on tissue homogenates prepared with protease inhibitors, corrected for total protein. Statistical comparison was by Wilcoxon signed rank pair analysis and Spearman's correlation.

Results 69 active UC patients (54 paired normal/inflamed mucosa) and 69 controls were compared. Significant elevation in IL-8 $(p<0.001$; $\mathrm{p}<0.001)$ and reduction in TGF $\beta(\mathrm{p}<0.02 ; \mathrm{p}<0.0002)$ with significant positive correlation of IL- $8(\mathrm{r} 2=+0.46 ; \mathrm{p}<0.01)$ and negative correlation of TGF $\beta(\mathrm{r} 2=-0.46 ; \mathrm{p}<0.01)$ to severity of inflammation was detected in inflamed compared with non-inflamed mucosa from the same patient and compared to age-sex matched control mucosa; however, TNF $\alpha$ concentration was not significantly different. Comparisons of macroscopically inflamed mucosa compared with non-inflamed mucosa from the same patients also demonstrated significant reduction in concentration of IFN $\gamma(p<0.001)$, IL-4 $(\mathrm{p}<0.005)$ and IL-17A $(\mathrm{p}<0.002)$. No significant differences were noted between normal tissue from UC patients and external controls. Conclusion Our findings suggest that IL-8 (a neutrophil chemoattractant) is elevated and TGF $\beta$ (involved in cell repair) is reduced with no change demonstrated for TNF $\alpha$. Significantly lower concentration of IFN $\gamma$, IL-4 and IL-17A suggests downregulation of Th1, Th2 and Th17 adaptive immune response. These findings suggest that the inflammatory response in UC may predominantly involve IL- 8 mediated neutrophil infiltration and failure of TGF $\beta$ mediated tissue healing, with limited evidence for the role of TNF $\alpha$ in mild-moderate distal UC.

Competing interests None declared.

\section{PMO-248 MUCOSAL UPREGULATION OF ARACHIDONIC ACID PRODUCTION IN ACTIVE ULCERATIVE COLITIS: DELIVERY OF PRO-INFLAMMATORY EICOSANOID PRECURSORS}

doi:10.1136/gutjnl-2012-302514b.248

${ }^{1} \mathrm{D}$ S Pearl, ${ }^{*} \mathrm{M}$ Masoodi, ${ }^{2} \mathrm{M}$ Eiden, ${ }^{3} \mathrm{~J}$ Brummer, ${ }^{3} \mathrm{D}$ Gullick, ${ }^{4} \mathrm{M}$ A Whittaker, ${ }^{4} \mathrm{H}$ Nitch-Smith, ${ }^{3} \mathrm{~J} \mathrm{~F}$ Brown, ${ }^{3} \mathrm{~J} \mathrm{~K}$ Shute, ${ }^{3} \mathrm{G}$ Mills, ${ }^{5} \mathrm{C}$ C Philip, ${ }^{1} \mathrm{~T}$ M Trebble. ${ }^{1}$ Gastroenterology Department, Portsmouth Hospitals NHS Trust, Portsmouth, UK; ${ }^{2}$ Elsie Widdowson Laboratory, Medical Research Council, Cambridge, UK; ${ }^{3}$ Institute of Biomedicine and Biomolecular Sciences, University of Portsmouth, Portsmouth, UK; ${ }^{4}$ Histopathology Department, Portsmouth Hospitals NHS Trust, Portsmouth, UK; ${ }^{5}$ Institute of Human Nutrition, School of Medicine, University of Southampton, Southampton, UK

Introduction Ulcerative colitis (UC) is a colonic inflammatory disorder of unconfirmed aetiology. Eicosanoids, inflammatory mediators involved in UC pathogenesis, are enzymatically converted from dietary polyunsaturated fatty acids (PUFA), arachidonic acid (AA) and eicosapentaenoic acid (EPA), themselves competitive 